Proceedings

\title{
Pitfalls of Accurate Protein Determination inside PLGA Nanoparticles Using the Micro BCA Assay ${ }^{\dagger}$
}

\author{
Marta Clerici ${ }^{1, *}$, Levi Collin Nelemans ${ }^{1,2}$, Matej Buzgo ${ }^{1}$ and Aiva Simaite ${ }^{1}$ \\ 1 InoCure s.r.o., R\&D Lab, Prumyslová 1960, 25088 Celákovice, Czech Republic; levi@inocure.cz (L.C.N.); \\ matej@inocure.vz (M.B.); aiva@inocure.cz (A.S.) \\ 2 Department of Hematology, University Medical Center Groningen, University of Groningen, \\ Groningen, $9713 \mathrm{AV}$, The Netherlands \\ * Correspondence: marta@inocure.cz \\ + Presented at the 1st International Electronic Conference on Pharmaceutics, 1-15 December 2020; \\ Available online: https://iecp2020.sciforum.net/.
}

Citation: Clerici, M.; Nelemans, L.C.; Buzgo, M.; Simaite, A. Pitfalls of Accurate Protein Determination Inside PLGA Nanoparticles Using the Micro BCA Assay. Proceedings 2021, 78, 28. https://doi.org/10.3390/ IECP2020-08671

Published: 1 December 2020

Publisher's Note: MDPI stays neutral with regard to jurisdictional claims in published maps and institutional affiliations.

Copyright: $(\odot 2020$ by the authors. Licensee MDPI, Basel, Switzerland. This article is an open access article distributed under the terms and conditions of the Creative Commons Attribution (CC BY) license (http://creativecommons.org/licenses/by/4.0/).

\begin{abstract}
Cancer is one of the leading causes of death in the world and protein therapeutics play an important role in combating this disease. Novel nanocarriers are needed for optimal delivery, enhanced therapeutic effect, and protection of proteins. Poly Lactic-co-Glycolic Acid (PLGA) nanoparticles are commonly used, since they are non-toxic, biodegradable, and allow for the sustained release of the active pharmaceutical ingredient (API). Accurate quantification of the therapeutic inside these nanocarriers is essential for further development and precise in vivo experiments, especially for determining the correct therapeutic dose. Bicinchoninic acid (BCA) assay is one of the most popular methods of protein quantification, known for its low sensitivity to common surfactants. However, large discrepancies between published results are often observed, with determined protein encapsulation efficiencies (EE) varying from 20 to $80 \%$. We investigate the interference of excipients or the combination of excipients, on accurate EE determination of PLGA nanoparticles using the micro BCA assay. The EE was determined using multiple methods: by measuring the un-encapsulated protein (indirect approach) and directly by extracting the protein using sodium hydroxide and dimethyl sulfoxide. We show differences between the methods, highlight the most common pitfalls, and show the importance of using correct standards in assessing EE.
\end{abstract}

Keywords: PLGA nanoparticles; micro BCA assay; encapsulation efficiency; protein encapsulation

\section{Introduction}

Cancer together with cardiac diseases is the main cause of death in the developed world [1]. Medical treatments of cancer remain mostly surgical and are often combined with chemotherapy, radiation therapy, and hormonal therapy that are harmful and invasive. Many different types of drug delivery systems (DDS) are being developed to reduce undesirable side effects of cancer therapeutics. However, the development of DDS with sustained release properties and effective pharmacological activity remains a great challenge [2]. Ideally, nanocarriers would increase the efficiency of drugs by targeted delivery of precise therapeutic doses and overcome the adversities by reducing side effects. However, such precision medicine is yet to be realized.

One of the properties needed for effective drug delivery is small particle size $(<200$ $\mathrm{nm}$ ) that would prevent their removal by spleen filtration and reticuloendothelial system [3]. However, the reduction of the size of carriers without compromising the drug loading and ensuring the predictable behavior of drugs, especially protein, is challenging [4]. Indeed, many factors, including the deleterious chemical and physical reactions during the in vitro studies may lead to erroneous estimation of active pharmaceutical ingredient (API) contents [5] and, subsequently, inaccurate dosing. Thus, methods that can allow 
rapid estimation of the encapsulation efficiency (EE) during the early stages of drug development are needed. Common pitfalls of EE estimations of protein encapsulated PLGA nanoparticles (NPs) are discussed in this work.

There are many methods to estimate the EE of the proteins in NPs, such as the biuret method [6], the Lowry method [7], the bicinchononic acid (BCA) assay [8], and the Coomassie dye binding, or Bradford assay [9] among others. The most common method characterizing the quantity of proteins in DDS is the BCA assay. The principle of this method is that proteins can reduce $\mathrm{Cu}^{+2}$ to $\mathrm{Cu}^{+1}$ in an alkaline solution (the biuret reaction) and result in a purple color formation by bicinchoninic acid. The reduction of copper is mainly caused by four amino acid residues including cysteine or cystine, tyrosine, and tryptophan that are present in protein molecules [10]. Indeed, BCA assay has been used in multiple publications to estimate the EE in NPs [5,11,12]. However, it seems that there is no universal consensus on the measurement protocol. Moreover, despite the simplicity of the assay, there is a high variance between obtained results even when similar formulations are used [11,13].

Multiple methods have been reported for the calculation of the EE of proteins. Some authors claim the EE only based on the indirect method of the un-encapsulated protein in the supernatant $[11,14,15]$ others destroy particles and estimate the EE directly $[11,13]$. However, even for the latter, multiple approaches have been reported. More specifically, PLGA particles can be broken down using dichloromethane [16,17], acetonitrile [18], sodium hydroxide ( $\mathrm{NaOH}$ ) [12,19], and dimethylsulfoxide (DMSO) [5]. However, very often apparent similar encapsulation methods, lead to differences in reported EE [11]. This raises the question, if the differences are caused by slight variations in the particle preparation protocols, or are there flaws in the estimation of EE?

In this paper we investigate and compare multiple methods to estimate the protein content in the PLGA-based NPs. We compare direct and indirect EE determination methods, and also results from direct EE obtained with two different methods, DMSO and $\mathrm{NaOH}$. We show that a detailed study of different EE quantification methods is crucial as protein concentration may be overestimated by neglecting the interference of the NPs to the BCA assay. The selection of the right quantification method is essential for measuring the quantity, activity, and the release rates of protein APIs.

\section{Experiments}

\subsection{Materials}

Throughout the work, the following materials were used:

For the nanoparticle (NPs) production, poly(lactic-co-glycolic acid), PLGA 5004 A (50:50) kindly provided as a gift by Corbion, ethyl acetate (EA) from VWR and poly (vinyl alcohol) (PVA) 5-88 was purchased from Sigma-Merck. The active ingredient used was bovine serum albumin (BSA) from PAA.

For measurement of encapsulation efficiency (EE): micro BCA assay Kit from Sigma Aldrich, sodium hydroxide ( $\mathrm{NaOH}$ ) from Penta, sodium dodecylsulfate (SDS) from VWR, dimethylsulfoxide (DMSO) from Applichem, and hydrochloric acid $(\mathrm{HCl})$ from SigmaAldrich.

\subsection{Methods}

\subsubsection{Preparation of PLGA NPs}

BSA-loaded PLGA NPs were prepared through a modified solvent emulsificationevaporation method based on a w/o/w double emulsion technique adapted from $[5,15,20]$. The protocol is the following: $100 \mathrm{mg}$ of PLGA 5004A was dissolved in $1 \mathrm{~mL}$ of EA. Then, $80 \mu \mathrm{L}$ of a $25 \mathrm{mg} / \mathrm{mL}$ BSA solution was added, and the polymeric solution was sonicated for $30 \mathrm{~s}$ with $70 \%$ of amplitude using the probe ultrasound (US) homogenizer (from Qsonica sonicators). After this, $4 \mathrm{~mL}$ of $2 \%$ PVA in distilled water, was added and the 
emulsion was mixed again by sonication. Finally, $7.5 \mathrm{~mL}$ of surfactant was added to the solution and the solvent was removed using a vacuum pump.

\subsubsection{Indirect Encapsulation Efficiency (IEE)}

The encapsulation efficiency was measured indirectly by, first, spinning down the particles for $25 \mathrm{~min}$ at 22,000 RCF in a Centurion Scientific Benchtop Centrifuge, and then, using the micro BCA assay to detect the amount of not encapsulated protein in the supernatant. IEE was calculated as a difference between the total amount of BSA used in the formulation and the free detected one.

Briefly, IEE was determined using the following equation:

$$
I E E \%=\frac{\text { total amount of BSA-free BSA in supernatant }}{\text { total amount of BSA }} \times 100 \text {. }
$$

\subsubsection{Direct Encapsulation Efficiency (DEE)}

The encapsulation efficiency was measured directly using two different methods: DMSO extraction [5] and $\mathrm{NaOH}$ extraction [11].

DMSO extraction: Following this method, particles were washed three times: particles were spun down at 22,000 RCF for $20 \mathrm{~min}$, the supernatant was collected, distilled water was added to the particles, and particles were resuspended using US probe at $20 \%$ amplitude for $3 \mathrm{~s}$ on and $5 \mathrm{~s}$ off (repeated three times). In order to verify the precision of this method, during the last washing step, unwashed particles were also spun down and then subjected to the same extraction as washed ones. This allowed us to measure the total amount of BSA, in the supernatant and inside the NPs. This value was then compared to the theoretical total volume of BSA added. After the last washing step, particles were left to dry at $50{ }^{\circ} \mathrm{C}$. When particles were dry (approx. $10 \mathrm{mg}$ of PLGA), $1.5 \mathrm{~mL}$ of DMSO was added and incubated for $1 \mathrm{~h}$ under constant agitation at room temperature. After $1 \mathrm{~h}$, when there was no visible pellet, the solution was mixed with $3.75 \mathrm{~mL}$ of $0.1 \mathrm{M} \mathrm{NaOH}$ and $0.05 \%$ SDS. To measure the amount of protein that was inside the particles, the micro BCA assay was used and for this a trendline with the DMSO/NaOH/SDS mixture was made. Blank NPs were used as a negative control, to check for potential interferences.

$\mathrm{NaOH}$ extraction: For the second method, particles were washed three times in distilled water: solutions with particles were spun down at 22,000 RCF for $20 \mathrm{~min}$, the supernatant was collected, and particles were resuspended with US probe at $20 \%$ amplitude (3 $\mathrm{s}$ on and $5 \mathrm{~s}$ off) three times. During the last washing step, instead of water, $950 \mu \mathrm{L}$ of 0.1 $\mathrm{M} \mathrm{NaOH}$ and $5 \%$ SDS were added and sonicated for $2 \mathrm{~min}$ at $20 \%$. Then, particles were incubated for $24 \mathrm{~h}$ at room temperature under continuous shaking. After $24 \mathrm{~h}$, when the particles were completely dissolved, $50 \mu \mathrm{L}$ of $2 \mathrm{M} \mathrm{HCl}$ was added to neutralize the solution and then, spun down at 10,000 RCF for $5 \mathrm{~min}$. The amount of protein in the solution was measured with the micro BCA assay. Two different trendlines were prepared for the micro BCA assay: one with only SDS and $\mathrm{NaOH}$ and another one also contained blank PLGA NPs which were subjected to the same treatment as particles with BSA inside. Blank NPs were used as the negative control, while blank NPs with BSA added at the end of the synthesis in a known concentration $(40 \mu \mathrm{g} / \mathrm{mL})$ were used as the positive control. For both methods, DEE was determined using the following equation:

$$
D E E \%=\frac{\text { Detected } B S A \text { in extraction }}{\text { total amount of } B S A} \times 100 .
$$

\subsubsection{Characterization}

The formulation was characterized regarding mean particle size and polydispersity index (PDI) using the Nanophox Dynamic Light Scattering (DLS) with photon cross-correlation spectroscopy from Sympatec. NPs were analyzed immediately after the synthesis. For DLS measurements, samples of blank and BSA-loaded PLGA NPs were prepared by taking a small amount $(100 \mu \mathrm{L}$ with $10 \times$ dilution) of the solution. All DLS experiments 
were carried out at $25^{\circ} \mathrm{C}$. The IEE and DEE were determined by UV-Vis spectroscopy using a microplate reader from SpectraMax. After adding the reagents of the micro BCA in a 96-well plate, it was incubated at $37^{\circ} \mathrm{C}$ for $2 \mathrm{~h}$ and, then, the adsorbance at $562 \mathrm{~nm}$ wavelength was measured. All experiments were done in triplicates. The reported values correspond to mean values with a standard deviation.

\section{Results}

A solvent emulsification-evaporation method based on a w/o/w double emulsion technique was used to produce blank NPs without protein as the negative control, BSAloaded NPs, and blank NPs with adsorbed BSA as the positive control. The BSA amount was then determined for all the particles using the direct and indirect BSA quantification. A schematic illustration of the preparation method is depicted in Figure 1a. As shown in Figure $1 \mathrm{~b}$, all prepared particles had a similar size below $250 \mathrm{~nm}$ that is in agreement with the value reported previously [20]. The significant differences obtained in the determination of BSA amount by indirect and direct methods are discussed in the following sections.



(a)

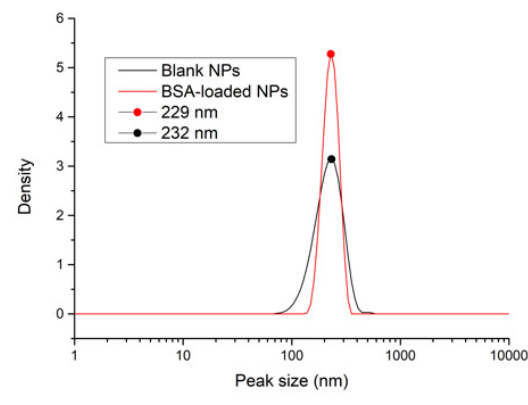

(b)

Figure 1. (a) Schematic representation of Poly Lactic-co-Glycolic Acid (PLGA) NPs synthesis; (b) particle size distribution of blank PLGA NPs and bovine serum albumin (BSA)-loaded PLGA NPs.

\subsection{Indirect Encapsulation Efficiency}

Using the indirect method, only very low amounts of BSA were detected in the supernatant. Consequently, the IEE \% calculated was very high, indicating that $88.77 \pm 0.03 \%$ of the protein was encapsulated inside the particles. There was no interference from the excipients in the NPs supernatant-negative control (blank NPs) did not show any absorbance. The results are summarized in Figure 2.

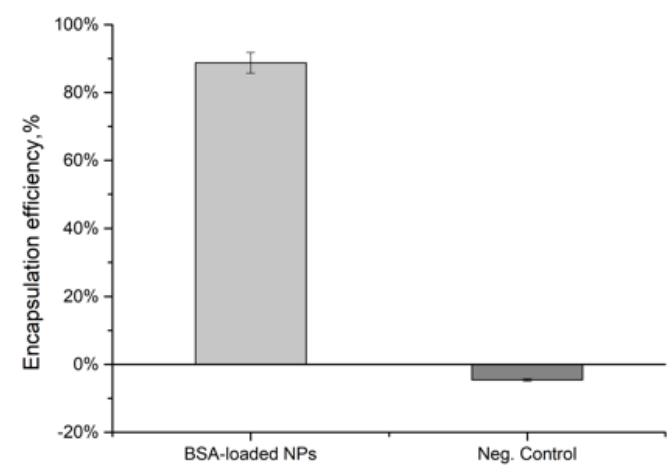

Figure 2. Indirect encapsulation efficiency (IEE) estimated by measuring the non- encapsulated protein inside the supernatant.

\subsection{Direct Encapsulation Efficiency}


Direct encapsulation efficiency was measured using two methods: with DMSO extraction and with $\mathrm{NaOH}$ extraction as reported in Section 2.2. The results of the two methods are summarized in this section.

\subsubsection{DMSO Extraction}

Firstly, experiments were done using the protein extraction by DMSO, commonly used to break down PLGA NPs [5]. The calibration curve (see Figure 3a) was made with the same ratio of $\mathrm{NaOH} / \mathrm{SDS} / \mathrm{DMSO}$, which was used for the NPs. Measured absorbance was lower than expected, since the highest concentration $(200 \mu \mathrm{g} / \mathrm{mL})$ did not even reach 1. In the following experiments, not washed NPs were used as positive control and the value was compared with the theoretical total amount of BSA added.

Using this detection method, significant interference from NPs was detected in both cases. As shown in Figure 3b, the calculated DEE exceeded 10\% for both washed and not washed blank NPs. Moreover, the detected total concentration was also lower than the theoretical one-only $76.17 \pm 0.007 \%$, while the calculated DEE was $62.33 \pm 3.51 \%$. However, given the high negative controls, this made the estimation unreliable.

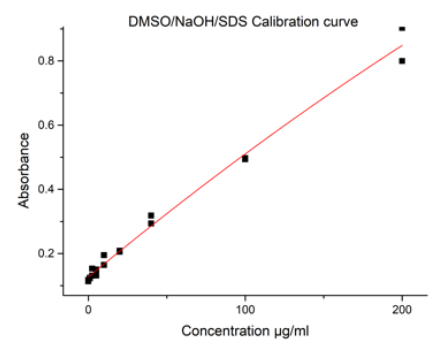

(a)

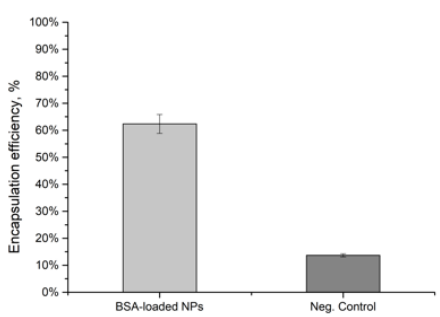

(b)

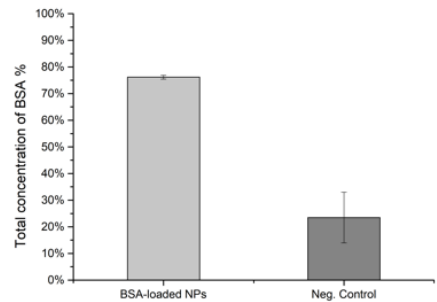

(c)

Figure 3. (a) Dimethylsulfoxide (DMSO)/NaOH/SDS calibration curve made for protein extraction by DMSO (b) direct $\mathrm{EE} \%$; (c) total concentration \% of BSA. In both cases $(\mathbf{b}, \mathbf{c})$, the negative control showed high interference.

\subsection{2. $\mathrm{NaOH}$ Extraction}

Several experiments were made to optimize the protocol, using different concentrations of $\mathrm{NaOH}(1 \mathrm{M}, 0.1 \mathrm{M}$, and $50 \mathrm{mM})$, different incubation time $(18,24$, and $48 \mathrm{~h})$ and temperature $\left(37^{\circ} \mathrm{C}\right.$ and room temperature). The trial with $50 \mathrm{mM}$ was made in order to avoid the neutralization step, which could cause faster hydrolysis of PLGA due to the high $\mathrm{pH}$ environment. However, in that case, particles were not fully dissolved after $24 \mathrm{~h}$. For the following experiments, extraction with $0.1 \mathrm{M} \mathrm{NaOH}$ for $24 \mathrm{~h}$ was used.

In this set of experiments, we used two trendlines for estimation of the DEE: trendline with nothing but the solutions used ( $5 \%$ SDS and $0.1 \mathrm{M} \mathrm{NaOH})$ and the trendline with the $10 \mathrm{mg} / \mathrm{mL}$ of blank NPs. Both trendlines are shown in Figure 4. Significant differences in adsorption intensity were observed between the trendlines. An almost two-fold reduction in adsorption for the same amount of the protein was observed when NPs were added and then broken down in the trendline for the BCA assay. It is apparent, that the encapsulation efficiency results that would be obtained using these two calibration curves will not be consistent. 


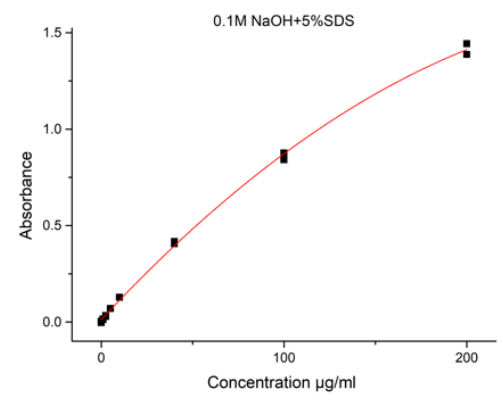

(a)

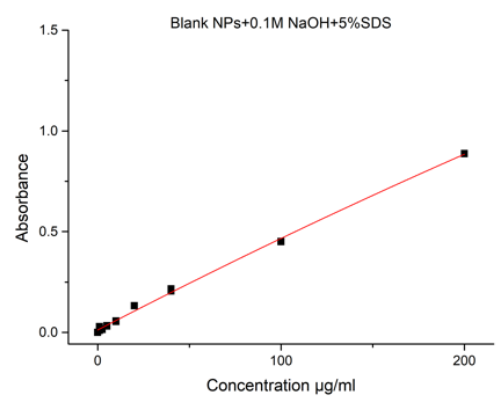

(b)

Figure 4. Trendlines used for $\mathrm{NaOH}$ extraction: (a) trendline made only with 5\% SDS and $0.1 \mathrm{M} \mathrm{NaOH}$; (b) trendline made also with blank NPs inside.

As previously, EE was measured for the particles without BSA (negative control), with BSA encapsulated, and with BSA added to blank NPs after particle preparation (positive control). The results were evaluated considering the two calibration curves. In both cases, the negative control of blank NPs showed BSA concentration about $0 \mu \mathrm{g} / \mathrm{mL}$, meaning that there was no interference of the broken down PLGA NPs during the BSA assay. However, there was a difference in the detected concentration of protein in the positive control. When the trendline made with NPs was used for calculations, the value was 31.76 $\pm 1.65 \mu \mathrm{g} / \mathrm{mL}$-close to the theoretical one $(40 \mu \mathrm{g} / \mathrm{mL})$. However, in the case of the trendline without NPs, the detected concentration was very low $-14.58 \pm 0.80 \mu \mathrm{g} / \mathrm{mL}-$ less than half of the theoretical value. The same was observed when the encapsulation efficiency in the NPs was calculated - two times with different values. As shown in Figure 5, according to the trendline made only with $\mathrm{NaOH}$ and SDS the $\mathrm{EE} \%$ is $29.77 \pm 0.001 \%$, on the other hand, considering the trendline made also with blank NPs the EE \% is $58.13 \pm 0.002 \%$.

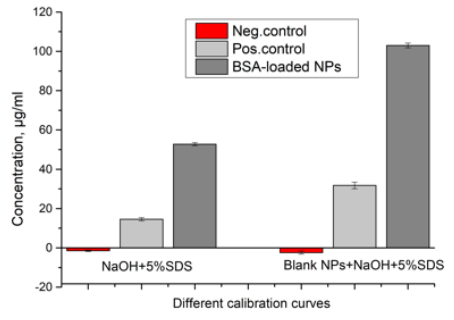

(a)

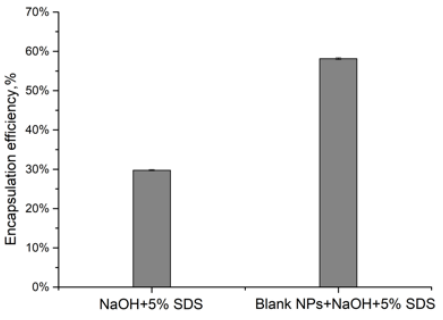

(b)

Figure 5. Results from $\mathrm{NaOH}$ extraction: (a) concentrations of negative and positive controls and BSA-loaded NPs detected using micro BCA assay; (b) difference between direct EE\% obtained using $\mathrm{NaOH}-\mathrm{SDS}$ trendline and the trendline with blank NPs.

\section{Discussion}

PLGA NPs are commonly considered to be a suitable carrier for protein-based therapeutics. However, that requires the ability to accurately estimate the physicochemical characteristics of PLGA based drug-delivery systems, including the EE and the loading capacity. One of the most common methods to characterize the EE-protein content quantification in the supernatant $[11,15,20]$. However, our results suggest that the measurement of the encapsulation efficiency with indirect and direct approaches, both using the micro BCA assay, may lead to significantly different results. Specifically, EE measured indirectly may overestimate the encapsulation efficiency. We have measured the encapsulation efficiency of $80 \%$, similar to the one previously reported [20], however, this value was not supported by the direct encapsulation efficiency measurements. 
The most alarming observation presented in this paper is the difference in the encapsulation efficiency measurement done with inappropriate standards. As we have shown, the use of the blank NPs in the standards is needed in order to accurately estimate the amount of the encapsulated protein (in this case, accuracy was assumed by having a close value of the positive control). We have observed a reduction in the absorbance due to the presence of the PLGA NPs inside the BCA reaction solution. Interestingly, there was no interference with the BCA reaction when the PLGA NPs were hydrolyzed separately and then mixed with the known amount of protein.

We have hypothesized that the interference arises from the polymer hydrolysis in presence of the protein and made multiple experiments trying to elucidate the mechanism of such reaction. We speculated that the process of PLGA hydrolysis that would lead to local acidification of the environment could also hydrolyze proteins adsorbed on the surface. We have tested if the phenomenon is time-dependent by using different concentrations of $\mathrm{NaOH}$ to control the rate of the reaction. As shown in Figure 6, the higher the concentration of $\mathrm{NaOH}$, the faster PLGA dissolves and so the higher the decrease in absorbance: as soon as PLGA is completely hydrolyzed, the absorbance does not change over time. However, experiments on the exposure of the particles and protein mixture to low $\mathrm{pH}(\mathrm{pH} 1-2)$ prior to the micro BCA assay, did not cause any interference. Our work demonstrates various pitfalls that may lead to inaccurate estimation of the EE. However, a clearer understanding of the mechanisms involved in the PLGA NPs interference to micro BCA assay is a subject of our future investigations.

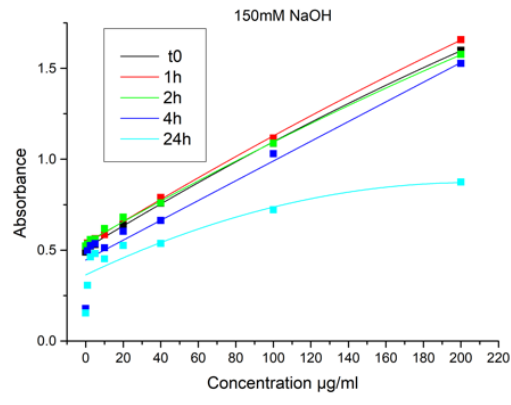

(a)

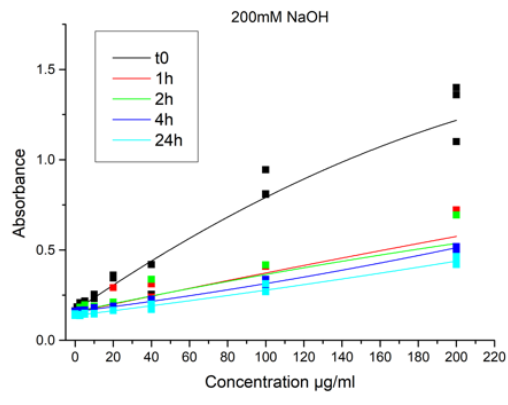

(b)

Figure 6. Calibration curves made with NPs and different concentrations of NaOH tested over time: (a) calibration curves made with $150 \mathrm{mM} \mathrm{NaOH}$; (b) calibration curve made with $200 \mathrm{mM} \mathrm{NaOH}$.

\section{Conclusions}

The choice of the right method to determine the real encapsulation efficiency of particles, and in this specific case of PLGA NPs, is extremely important in order to have a correct estimation of the amount of drug present inside and that has to be released for the medical treatment. In this work, we have shown that there is a significant difference in EE determination using three common methods: indirect measurement, direct measurement after $\mathrm{NaOH}$ extraction, and after DMSO extraction. Our results suggest that correct controls need to be chosen to avoid overestimation of the EE, as polymer excipients in the solution may cause interference, even when negative controls do not display them.

\section{Abbreviations}

PLGA: Poly lactic-co-glycolic acid; API: Active pharmaceutical ingredient; NPs: Nanoparticles; BCA: Bicinchoninic acid; EE: Encapsulation efficiency; NaOH: Sodium hydroxide; DMSO: Dimethylsulfoxid; EA: Ethyl acetate; PVA: Poly (vinyl alcohol); BSA: Bovine serum albuminin; SDS: Sodium dodecylsulfate; $\mathrm{HCl}$ : Hydrochloric acid; IEE: Indirect encapsulation efficiency; DEE: Direct encapsulation efficiency. 
Author Contributions: M.C., L.C.N., A.S., and M.B. conceived and designed the experiments; M.C. performed the experiments; M.C. analyzed the data; M.C., A.S., and L.C.N. wrote the paper. All authors have read and agreed to the published version of the manuscript.

Institutional Review Board Statement: Not applicable.

Informed Consent Statement: Not applicable.

Data Availability Statement: Not applicable.

Acknowledgments: This project has received funding from the European Union's Horizon 2020 research and innovation programme under the Marie Sklodowska-Curie grant agreement No 813871. This work was supported by European Union's Horizon 2020 research and innovation programme under the Marie Skłodowska-Curie grant agreements No. 823981 (actiTOX) and No.

824007 (iP-Osteo)

Conflicts of Interest: The authors declare no conflict of interest.

\section{References}

1. Neuse, E.W. Synthetic polymers as drug-delivery vehicles in medicine. Met.-Based. Drugs 2008, 2008, 1-19, doi:10.1155/2008/469531.

2. Pavlov, A.M.; Saez, V.; Cobley, A.; Graves, J.; Sukhorukov, G.B.; Mason, T.J. Controlled protein release from microcapsules with composite shells using high frequency ultrasound-Potential for in vivo medical use. Soft Matter 2011, 7, 4341-4347, doi:10.1039/c0sm01536a.

3. Hoshyar, N.; Gray, S.; Han, H.; Bao, G. The effect of nanoparticle size on in vivo pharmacokinetics and cellular interaction. Nanomedicine 2016, 11, 673-692, doi:10.2217/nnm.16.5.

4. Singh, N.; Joshi, A.; Toor, A.P.; Verma, G. Drug delivery: Advancements and challenges. In Nanostructures for Drug Delivery; Elsevier: Amsterdam, The Netherlands, 2017; pp. 865-886, doi:10.1016/B978-0-323-46143-6.00027-0.

5. Sah, H. A new strategy to determine the actual protein content of poly(lactide- co-glycolide) microspheres. J. Pharm. Sci. 1997, 86, 1315-1318, doi:10.1021/js960363q.

6. Gornall, A.G.; Bardawill, C.J.; David, M.M. Determination of serum proteins by means of the biuret reaction. J. Biol. Chem. 1949, $177,751-766$.

7. Kresge, N.; Simoni, R.D.; Hill, R.L. The Most Highly Cited Paper in Publishing History: Protein Determination by Oliver H. Lowry. J. Biol. Chem. 2005, 280, e26-e28, doi:10.1016/S0021-9258(20)56859-2.

8. Smith, P.; Krohn, R.; Hermanson, G.; Mallia, A.; Gartner, F.; Provenzano, M.; Fujimoto, E.; Goeke, N.; Olson, B.; Klenk, D. Measurement of protein using bicinchoninic acid. Anal. Biochem. 1985, 150, 76-85, doi:10.1016/0003-2697(85)90442-7.

9. Bradford, M.M. Determinación de proteínas: Método de bradford. Anal. Biochem. 1976, 72, 248-254.

10. He, F. BCA (Bicinchoninic Acid) Protein Assay. Bio-Protocol 2011, doi:10.21769/bioprotoc.44.

11. Amini, Y.; Jamehdar, S.A.; Sadri, K.; Zare, S.; Musavi, D.; Tafaghodi, M. Different methods to determine the encapsulation efficiency of protein in PLGA nanoparticles. Biomed. Mater. Eng. 2017, 28, 613-620, doi:10.3233/BME-171705.

12. Rosas, J.; Bayat, A.; Gascón, A.; Igartua, M.; Guzman, F.; Patarroyo, M.A.; De Castro, M. Biodegradable PLGA microspheres as a delivery system for malaria synthetic peptide SPf66. Vaccine 2001, 19, 4445-4451, doi:10.1016/S0264-410X(01)00192-X.

13. Zheng, C.H.; Liang, W.Q.; Yu, H.Y.; Chen, H.L. Evaluation of different methods to determine the loading of proteins in PLGA microspheres. Pharmazie 2004, 59, 232-233.

14. Gref, R.; Quellec, P.; Sanchez, A.; Calvo, P.; Dellacherie, E.; Alonso, M.J. Development and characterization of CyA-loaded poly(lactic acid)-poly(ethylene glycol)PEG micro- and nanoparticles. Comparison with conventional PLA particulate carriers. Eur. J. Pharm. Biopharm. 2001, 51, 111-118, doi:10.1016/S0939-6411(00)00143-0.

15. Fonte, P.; Soares, S.; Sousa, F.; Costa, A.; Seabra, V.; Reis, S.; Sarmento, B. Stability study perspective of the effect of freezedrying using cryoprotectants on the structure of insulin loaded into PLGA nanoparticles. Biomacromolecules 2014, 15, 3753-3765, doi:10.1021/bm5010383.

16. Yang, Y.Y.; Chung, T.S.; Ng, N.P. Morphology, drug distribution, and in vitro release profiles of biodegradable polymeric microspheres containing protein fabricated by double-emulsion solvent extraction/evaporation method. Biomaterials 2001, 22, 231-241, doi:10.1016/S0142-9612(00)00178-2.

17. Castellanos, I.J.; Cruz, G.; Crespo, R.; Griebenow, K. Encapsulation-induced aggregation and loss in activity of $\gamma$-chymotrypsin and their prevention. J. Control. Release 2002, 81, 307-319, doi:10.1016/S0168-3659(02)00073-1.

18. Diwan, M.; Park, T.G. Pegylation enhances protein stability during encapsulation in PLGA microspheres. J. Control. Release 2001, 73, 233-244, doi:10.1016/S0168-3659(01)00292-9.

19. Lam, X.M.; Duenas, E.T.; Daugherty, A.L.; Levin, N.; Cleland, J.L. Sustained release of recombinant human insulin-like growth factor-I for treatment of diabetes. J. Control. Release 2000, 67, 281-292, doi:10.1016/S0168-3659(00)00224-8.

20. Sousa, F.; Cruz, A.; Fonte, P.; Pinto, I.M.; Neves-Petersen, M.T.; Sarmento, B. A new paradigm for antiangiogenic therapy through controlled release of bevacizumab from PLGA nanoparticles. Sci. Rep. 2017, 7, 1-13, doi:10.1038/s41598-017-03959-4. 